Torreón Universitario

www.faremcarazo.unan.edu.ni / ISSN 2410-5708 / e-ISSN 2313-7215

Año 8 | Núm. 23 | pág. 6 - pág. 26 | octubre 2019 - enero 2020
Esta obra está bajo una licencia de Creative Commons

Atribución-NoComercial-SinDerivar 4.0 Internacional

\title{
Fallas estructurales de la oración subordinada adjetiva en la redacción del ensayo argumentativo
}

Dr. Álvaro Escobar Soriano

Docente investigador

UNAN-Managua

alvess007@gmail.com

MSc. Armando José Sandoval

Departamento de Español

UNAN-Managua

sandovalarmando7@gmail.com

Fecha de recepción: 20 de agosto, 2019 / Fecha de aceptación: 20 de noviembre, 2019

https://doi.org/10.5377/torreon.v8i23.9524

Palabras claves: errores, gramática, redacción, subordinada

adjetiva, ensayo argumentativo

\section{RESUMEN}

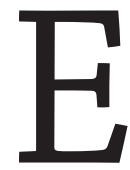

$\mathrm{n}$ el trabajo se aborda la redacción en el cual se validó el proyecto didáctico sobre el uso de la oración subordinada adjetiva como mecanismo de progresión temática en el ensayo argumentativo. La metodología implementada en la investigación es el análisis de un texto argumentativo diagnóstico. En ella, se detectó las carencias de conocimiento gramatical, en especial de la oración subordinada adjetiva. Esto se acompañó de una guía de observación, del llenado de encuesta y entrevista a los discentes seleccionados. Los resultados demostraron que los estudiantes tienen dificultades para expresarse por escrito. En parte por la desvinculación de la enseñanza de la gramática con la expresión escrita. 


\section{INTRODUCCIÓN}

El presente trabajo se enfoca en la expresión escrita. De forma directa en la redacción del ensayo argumentativo y en la funcionalidad de la gramática para la adquisición de esa macrohabilidad, como lo es la oración subordinada adjetiva. Este planteamiento se origina a partir de una prueba diagnóstica aplicada a estudiantes de primer año de la carrera de Derecho en 2015 y la reflexión realizada sobre la funcionalidad de la gramática para los usuarios del español. Esto promovió la búsqueda de trabajos relacionados con las dos temáticas.

Sin embargo, los hallazgos expresaban una preocupación por las dificultades en los estudiantes para dominar la expresión escrita. Todos hacían planteamientos generales sobre la problemática, las medidas de solución y dentro de sus conclusiones exponían que es el docente el indicado para desarrollar habilidades en el discente y una de ellas, la escritura. Por otra parte, ninguna de las tesis y artículos consultados consideró retomar en sus estudios de manera integrada la gramática y la expresión escrita. Por eso, es un estudio novedoso, porque combina la oración subordinada adjetiva como mecanismo de progresión temática en la redacción del ensayo argumentativo.

Por lo anterior, la importancia de este trabajo radica en buscar la solución a los problemas de escritura desde una gramática aplicada, funcional y útil para el discente. Con el sentido de que el estudiante asocie sus dificultades escriturales a la falta de dominio teórico-práctico del conocimiento de gramática. Por eso, el objetivo principal de este trabajo de investigación, es validar en la escritura del ensayo argumentativo, el uso de la oración subordinada adjetiva como mecanismo de progresión temática. De manera puntual, en este primer artículo se recogen los resultados relacionados al primer objetivo específico de investigación que consiste en describir las dificultades de los estudiantes de primer año de la carrera de Lengua y Literatura en el uso de los esquemas gramaticales en la producción escrita de un texto diagnóstico de tipo argumentativo.

\section{FUNDAMENTACIÓN TEÓRICA}

La teoría que sustenta el trabajo es la relacionada primeramente con la psicología del aprendizaje. Este es el punto de partida o de llegada de todo docente. Además, es este que debe de ocuparse del proceso que cada estudiante pone en práctica (Rodríguez y Moreira 2002). Por lo tanto, esto conlleva a realizar una redimensión del proceso de enseñanza, considerando hacer efectivo el aprendizaje. En este sentido el aprendizaje no se forma con la simple transmisión de conocimiento o el almacenamiento de este. (Chadwick, 1999).

Por otra parte, es real que el ser humano vive en proceso de crecimiento y de aprendizaje, el segundo es el primer modificador del comportamiento y del conocimiento. Al respecto Piaget (s. f.) afirma que "la concepción de un organismo plástico, modificado constantemente por 
el aprendizaje, por las influencias exteriores, por el ejercicio o la "experiencia" en el sentido empirista de la palabra" (p. 43). Es evidente que el estudiante vive en las aulas de clase un proceso constante, pero este solo es efectivo si recibe la atención adecuada usando estrategias proactivas para el aprendizaje, el cual modificaría positiva al alumno.

Por eso, se asume el enfoque constructivista, porque el aprendizaje debe ser producto de un proceso vivido por el discente. Una pregunta importante dentro de esta reflexión, como partida, qué es el constructivismo, para Hernández (2008) "La teoría constructivista se enfoca en la construcción del conocimiento a través de actividades basadas en experiencias ricas en contexto" (p.1). Son las actividades que enriquecen a los estudiantes, las que le dan experiencia. Si estas se toman de situaciones reales del contexto. Estos tendrían un verdadero aprendizaje o serían los constructores de sus conocimientos. Por eso, la teoría toma dentro de su base el enfoque comunicativo, porque el discente debe dominar su sistema de lengua y además, ser capaz de emplearlo en su contexto de forma efectiva (Luzón y Soría 1999).

En ese sentido, para que la enseñanza logre su efectividad debe contar con un diseño de estrategias de aprendizaje. Viva (2010) los define "como toda actividad o conjunto de actividades que posibilitan el aprendizaje y el autocontrol del mismo por parte de la persona que aprende" (p. 2). Es importante señalar que la garantía de la enseñanza dependerá en gran medida del conjunto de actividades que sean parte de la estrategia elaborada para tal fin. En la actualidad, son tanto los modelos de estrategia que se pueden consultar, o incluso existe la posibilidad de que el docente diseñe su propia estrategia de acuerdo con su situación educativa y características del grupo de estudiantes.

El producto de escritura que se busca es la redacción del ensayo argumentativo. Pero, qué es el ensayo argumentativo. Torres (2004) expresa que "El ensayo argumentativo puede definirse como un tipo de texto estructurado y unificado alrededor de una tesis que se sustenta de diversas formas como razones o ilustraciones" (p. 98-99). De forma oral el ser humano vive argumentando, defendiendo una postura, sin embargo, por escrito no logra concretar ideas coherentes que refuercen su punto de vista. Un aspecto importante, es que se retoma la redacción de forma procesal. Esto se empieza a lograr cuando la persona da pasos en el borrador mental incluyendo el proceso y el contenido del texto. (Meneses, Mata y Ravelo, 2007).

Finalmente, la oración subordinada adjetiva y esta desde el Manual de la nueva gramática (2010) "Se denominan oraciones subordinadas de relativo (también oraciones relativas o simplemente relativas) las encabezadas por un pronombre, adverbio o determinante relativo, o bien por los grupos sintácticos que estas voces forman" (p. 836). En este sentido, hoy se denomina oración subordinada de relativo las que son introducidas por los relativos: que, cual, 
quien, cuyo, cuya. Se entiende que es práctica, pero para el estudiante es un proceso complejo, porque no logra insertarla dentro de la oración, la cual representa la idea en el párrafo.

En ese sentido, la utilidad de la subordinación en la expresión escrita se fundamenta en la premisa de ampliar la idea principal. Por su parte, Giammatteo (2013) afirma que "Esta idea de continuidad se acentúa con la subordinada" (p. 12). Toda persona que domina su lengua materna debe conocer el beneficio del uso de la subordinación en sus escritos y dominar su construcción, porque de esa forma garantizará expresarse de forma fluida tanto oral como escrita. Rodríguez (2012) observa que es preciso hacer un trabajo con mayor profundidad en la enseñanza de la gramática, para dar cuenta de los fenómenos de construcción de un objeto gramatical, en especial de la oración subordinada.

\section{MATERIALES Y MÉTODOS}

El paradigma con que se trabajó la presente investigación es el sociocrítico, porque este propone dinamismo a este proceso. Además, es elemental que el docente sea parte activa de la investigación que se incluya en la práctica y con la teoría. Es la forma consciente de generar una investigación desde el interior del problema para darle solución. El tipo de investigación que se realizó es la llamada investigación-acción. En ese sentido, el docente desde su práctica realiza, ajusta e innova estrategias que permiten salir de los esquemas tradicionales, con el fin de dar solución a las problemáticas existente en las aulas de clase que dificultan el desarrollo del discente.

El enfoque utilizado para realizar la investigación fue el cualitativo. Esto permitió hacer desde el inicio del proceso, las descripciones del fenómeno sobre todos los elementos que intervienen en la producción escrita. Además, se hizo uso de técnicas cuantitativas, las que cumplieron función auxiliar, para presentar la realidad del fenómeno. Mediante la aplicación del enfoque estudio de caso se realiza una organización de la información obtenida, con carácter unitario, porque es a partir de este punto, que se puede analizar los trabajos individuales como un todo (Arzaluz, 2005).

La población para este estudio fueron los estudiantes de primer año de la carrera de Lengua y Literatura Hispánicas de la modalidad por encuentro (sabatino) de la UNAN-Managua, porque ellos serán docentes de secundaria en el futuro. Por lo tanto, la participación en la aplicación de la estrategia les formó conciencia en el arte de escribir.

El instrumento utilizado fue la redacción del texto argumentativo diagnóstico, a través de este se detectaron los problemas que enfrentan los estudiantes en la producción escrita, en especial en la redacción del ensayo argumentativo. Además, se confirmó que los estudiantes tienen algunas carencias gramaticales, que interfieren en su expresión escrita. Otra fue la guía 
de observación a los estudiantes durante la escritura del texto diagnóstico argumentativo. Por lo tanto, es una forma de estar cara a cara con las incidencias en el aula de clase, con el problema y sus aportes, los cuales serán incluidos en el análisis de los datos.

\section{RESULTADOS Y DISCUSIÓN}

En un primer acercamiento al conocimiento de gramática, se les consultó a través de la lista cotejo dos aspectos fundamentales, a los discentes:

Utilizó la gramática

- Sin gramática podría escribir

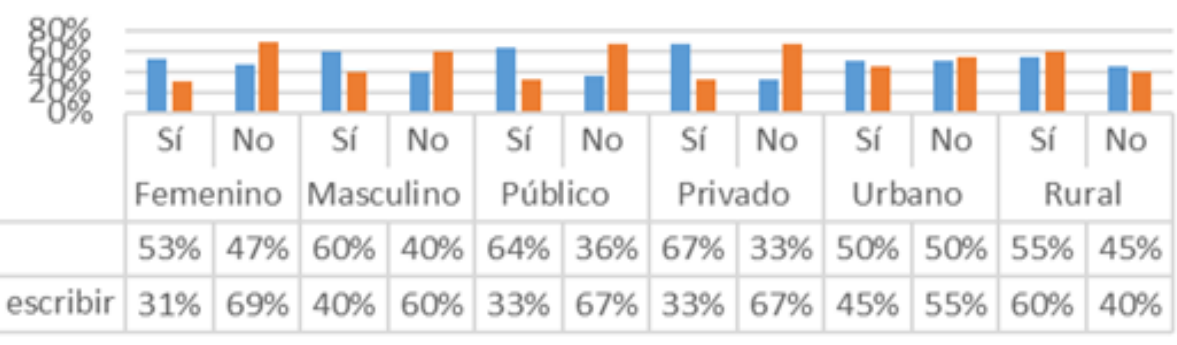

\section{Gráfico $\mathrm{N}^{\circ}$ 7. Lista de cotejo utilizada para el análisis del texto diagnóstico.}

El primer aspecto es el referido al uso de la gramática en la escritura. En el gráfico No 7 , la casilla "No", muestra porcentajes impactantes, femenino 47\%, público $36 \%$ y urbano $50 \%$, aseguran que la gramática no fue utilizad en el texto diagnóstico escrito. Este aspecto, es básico para esta investigación. Con la pregunta anterior se les consultaba sobre la importancia de la gramática para la escritura del texto, pero con la respuesta se infiere que ellos no conectan el conocimiento gramatical con la producción escrita y no reconocen la influencia de esta.

Los discentes, organizados según las variables, consideran no utilizar la gramática para escribir. No reconocen, que esta le da el conocimiento sobre su lengua materna y la posibilidad de estructurar ideas de manera cohesionadas y coherentes.

El segundo aspecto es una reflexión: podrían escribir sin la gramática. En el gráfico Nº7, se observa que la casilla del "Sí”, presenta porcentajes notorios (masculino 40\%, público 33\% y rural $60 \%)$. Esto reafirma el desconocimiento del uso de la gramática en la escritura. Los porcentajes expresan que sin la teoría gramatical se podría escribir el texto diagnóstico argumentativo. Es una realidad, existe una desvinculación entre la enseñanza de la gramática y la expresión escrita. Porque en la secundaria, se enseña gramática estructural, básicamente para el análisis sintáctico de oraciones aisladas.

Con la información obtenida mediante la lista de cotejo (gráfico No 7). Se tiene un primer acercamiento al estudiante y su conocimiento gramatical. Los segundos se forman con los estudios académicos, este a su vez se transforma en esquemas cognitivos, que finalmente se traducen en competencias. Pero, si ese proceso no se da los resultados son catastróficos. 
La primera categoría abordada en los gráficos $\mathrm{N}^{\circ} 8,9$ y 10, está relacionado con el uso del enunciado simple. Los porcentajes son altos: femenino $75 \%$, privado $83 \%$ y urbano $82 \%$, quienes indican utilizarlo en el escrito:

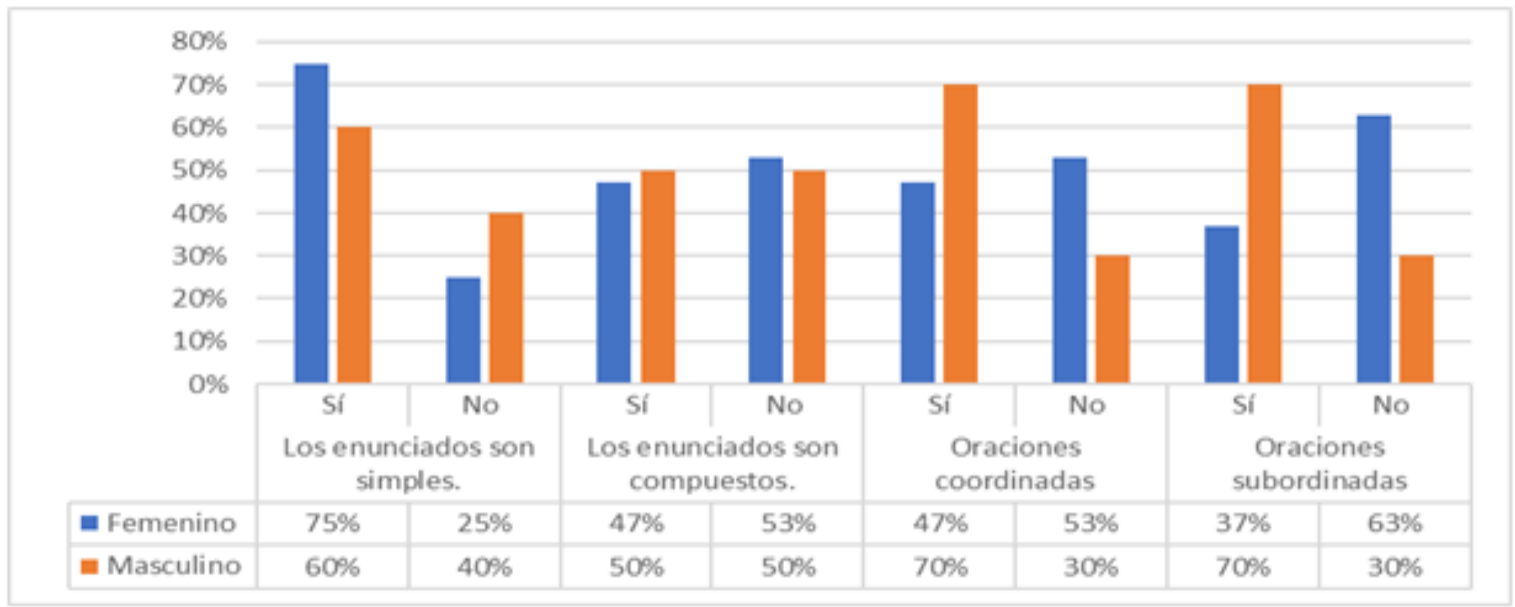

Gráfico $\mathrm{N}^{\circ}$ 8. Cuestionario utilizado para la revisión del texto diagnóstico. Fuente: tabla de análisis. ver anexo $\mathrm{N}^{\circ} 3.2$.

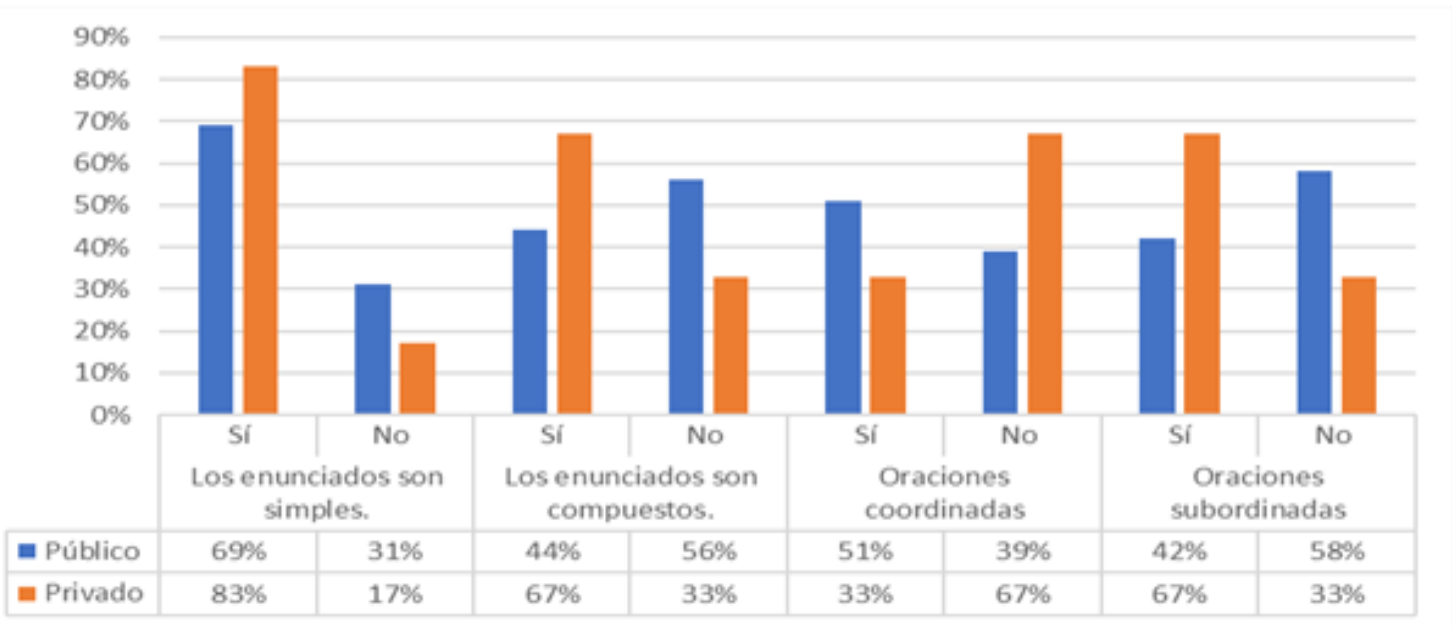

Gráfico $\mathrm{N}^{\circ}$ 9. Cuestionario utilizado para la revisión del texto diagnóstico. Fuente: tabla de análisis, ver anexo $\mathrm{N}^{\circ} 3.2$. 


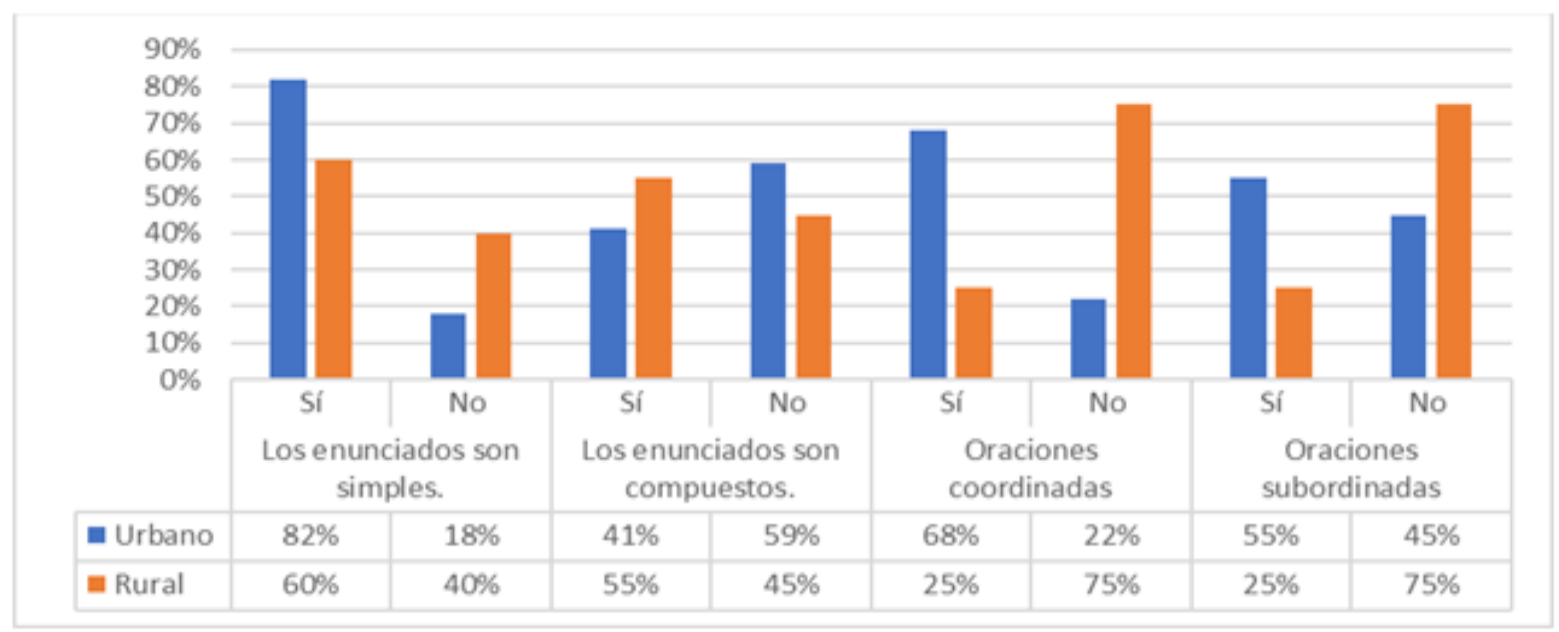

\section{Gráfico $\mathrm{N}^{\circ} 10$. Cuestionario utilizado para la revisión del texto diagnóstico. Fuente: tabla de análisis, ver anexo $\mathrm{N}^{\circ} 3.2$.}

Los textos TDA9 y TDA16, muestran una realidad distinta a la señalada en los gráficos $\mathrm{N}^{\circ} 8,9$ y 10. Los dos son ejemplos de enunciados, pero no simple, porque estos tienen más de una forma verbal conjugada y la teoría gramatical (Manual de la nueva gramática, 2008) indica que el enunciado simple se construye con una forma verbal conjugada. Esto es más que evidente, los discentes confunden el enunciado simple con el compuesto.

Por lo anterior, se puede decir que el porcentaje expresado en la casilla de "Sí" (masculino $50 \%$, privado 67\% y rural 55\%), de la segunda categoría de los gráficos No 8, 9 y 10 es acertado. Pero, con el resultado del análisis de los textos TDA9 y TDA16, este se encuentra por debajo de la realidad, porque el enunciado con mayor presencia en los textos diagnóstico, es el enunciado compuesto.

La tercera categoría mostrada en los gráficos $\mathrm{N}^{\circ} 8,9$ y 10 , está relacionada con el uso de la oración coordinada. Esta muestra resultados en la casilla de "Sî", que marcan una clara división: los grupos femenino (47\%), privado (33\%) y rural (25\%) tienen bajo porcentaje de uso, en cambio los grupos masculino (50\%), público (51\%) y urbano (68\%) alto porcentaje de uso de la oración coordinada. Esta partición reflejada en el conocimiento gramatical sobre los tipos de enunciados compuestos, es interesante, porque no hay criterios de unidad alrededor de este conocimiento. 
TDA 9

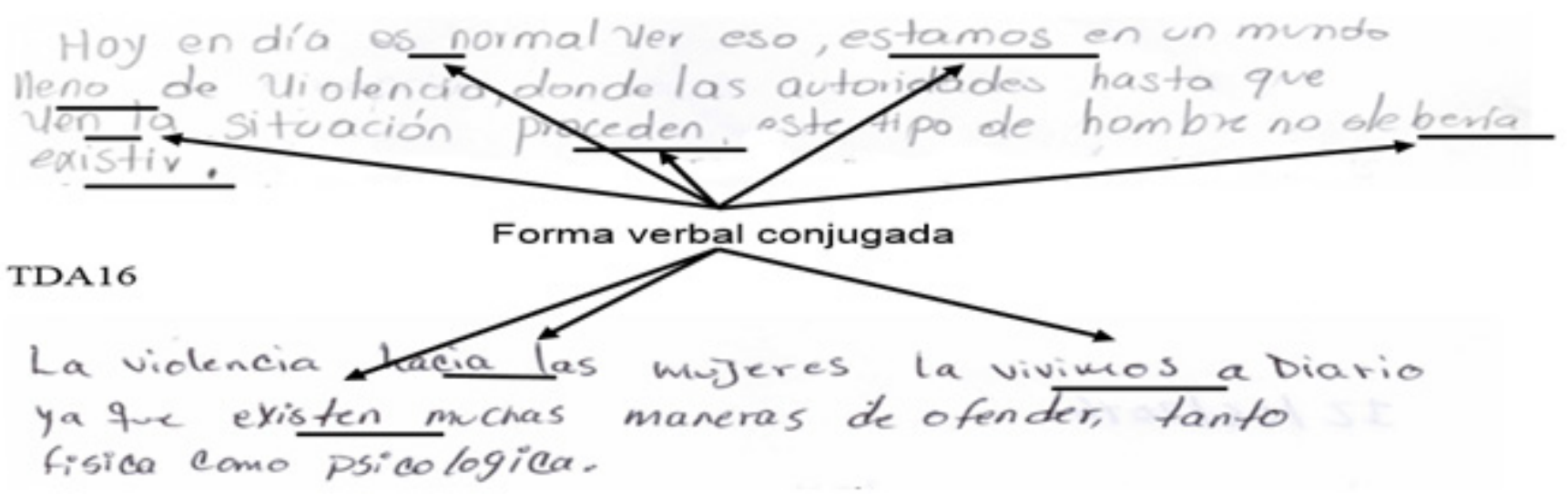

Sin embargo, la respuesta es un aspecto, el otro aspecto se completa con la revisión. En los textos TDA21 y TDA23 se observa esa particularidad. En el primero, la ausencia del enunciado coordinado y en el segundo la presencia de este. Es en este último, se puede ver que la coordinación es de tipo copulativa, construida con el nexo "y". Según la nueva gramática la coordinación homocategorial se produce cuando hay coordinación de elementos del mismo nivel sintáctico, pero en el texto no se produce este tipo de coordinación.

TDA21

Has mojidio es más qee ona violenciue hacia Al equal que todas las cosas, este también tor nosotnas mis qe podñan ser inapersibidos en nuestros mismas, inicice desde nuestras casas familias. propios hogares, con nuestras propios

TDA23

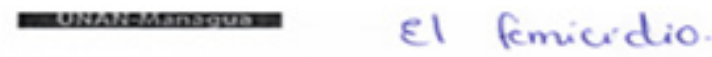

Hoy en da en tods el mundo thay muchos malta bs

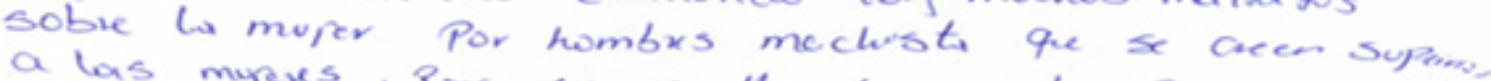

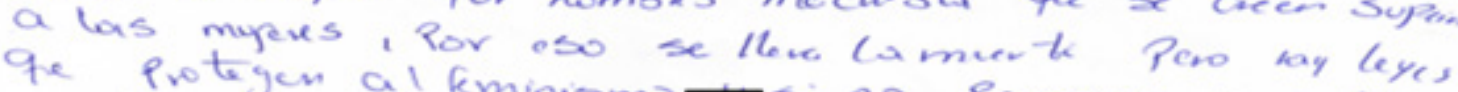
a este knomeno al kminiomotrsino. Poremos en a 110 Aiene que tener libe dorgivin disguncen" Por eso ca mare. importar la soledad ducise Por somisms, Sin Protige de manew segerayes ytener en cuente fe nos un alla a esto segura y somos capans de dea a esto. Rana póder vivir en Riz. 
La última categoría presente en los gráficos $\mathrm{N}^{\circ}$ 8, 9 y 10 aborda un elemento importante para esta investigación, este es el referido a la oración subordinada. Los porcentajes reflejados en la variable masculino $70 \%$, privado $67 \%$ y urbano $55 \%$ afirman haber utilizado este tipo de oración compuesta. Los resultados son alentadores, porque los estudiantes en teoría conocen de la oración mencionada.

TDA23

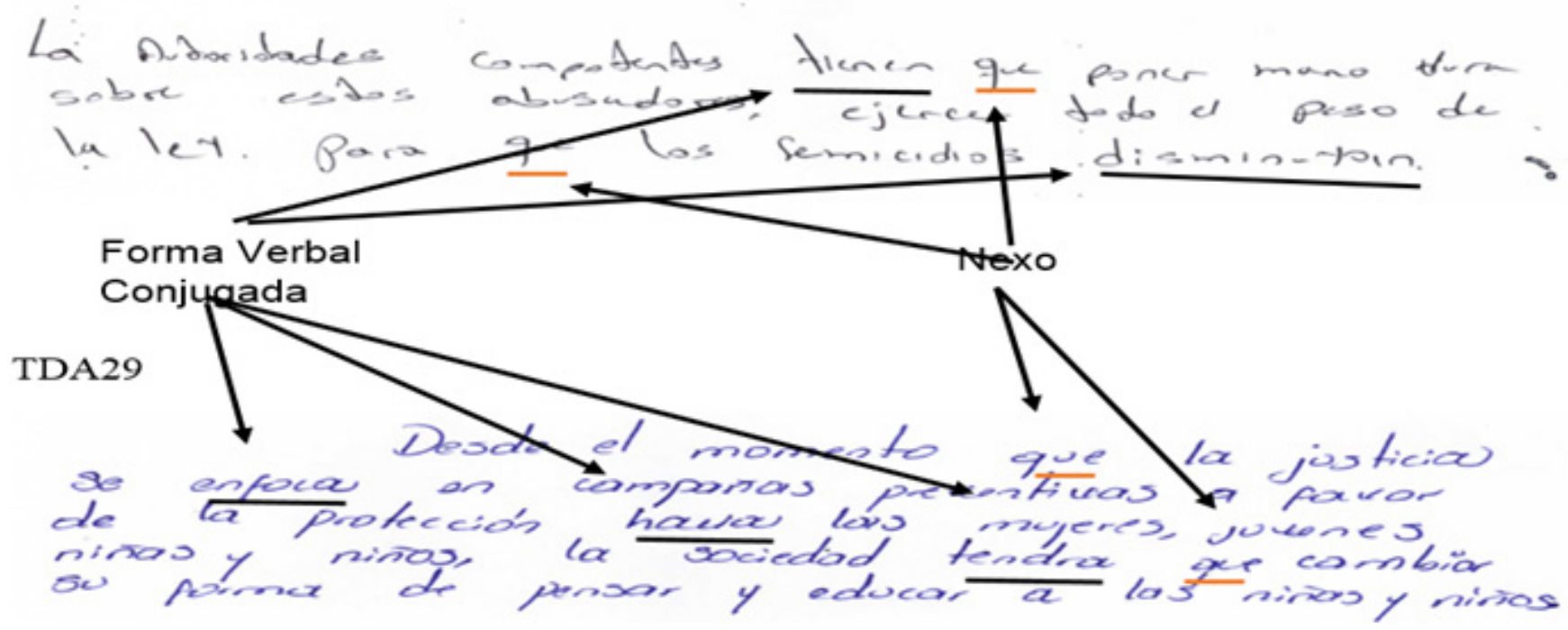

En los textos TDA23 y TDA29, se identifica la presencia de la oración subordinada. Primeramente, porque en el enunciado se identifica más de una forma verbal conjugada; segundo el uso del relativo "que". Estos dos elementos son básicos para la existencia de una oración compuesta, de tipo subordinada. Un aspecto a destacar es que este tipo de estructura está presente en la mayoría de los textos diagnósticos. Sin embargo, los discentes no lograron afirmar en su totalidad, la presencia de este tipo de oración en el escrito. 


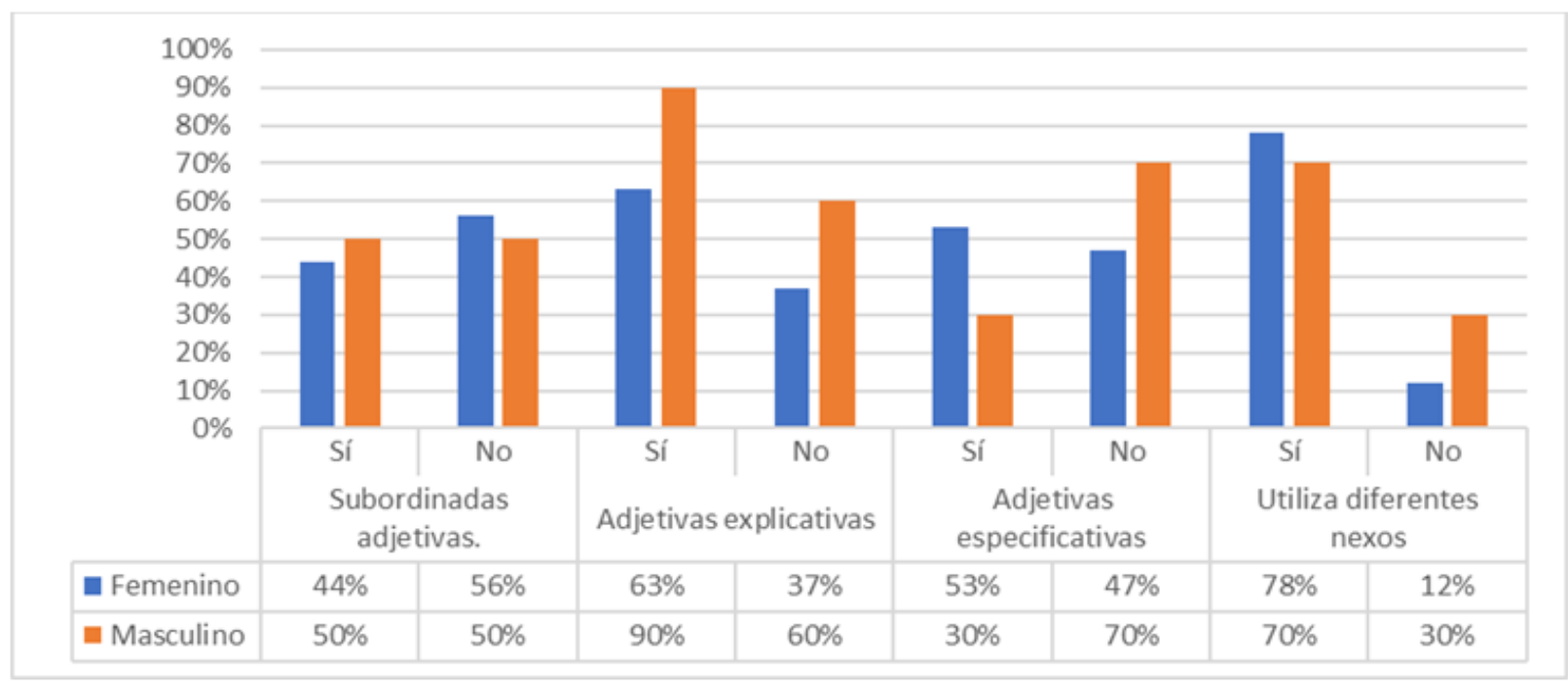

Gráfico $\mathrm{N}^{\circ} 11$. Cuestionario utilizado para la revisión del texto diagnóstico. Fuente: tabla de análisis, ver anexo No3.4.

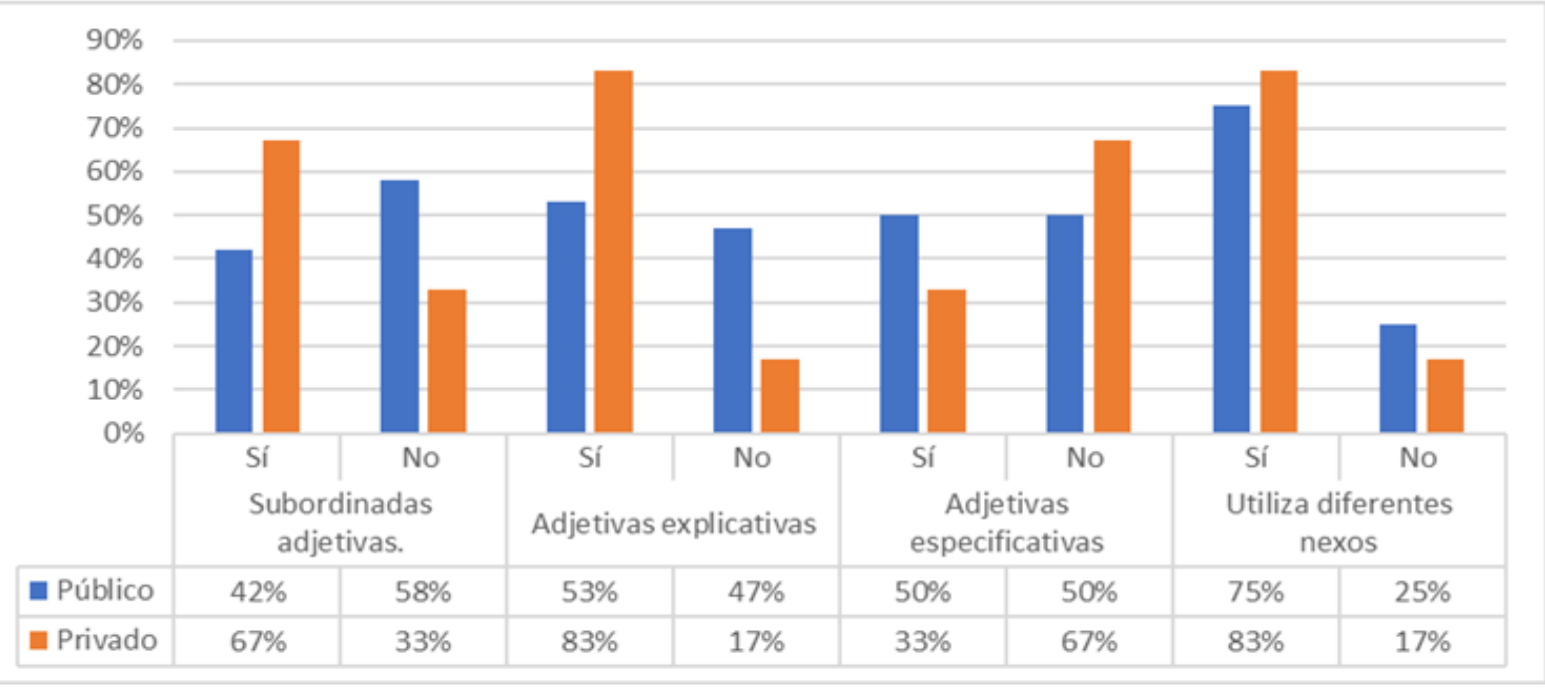

Gráfico $\mathrm{N}^{\circ}$ 12. Cuestionario utilizado para la revisión del texto diagnóstico. Fuente: tabla de análisis, ver anexo $\mathrm{N}^{\circ} 3.4$. 


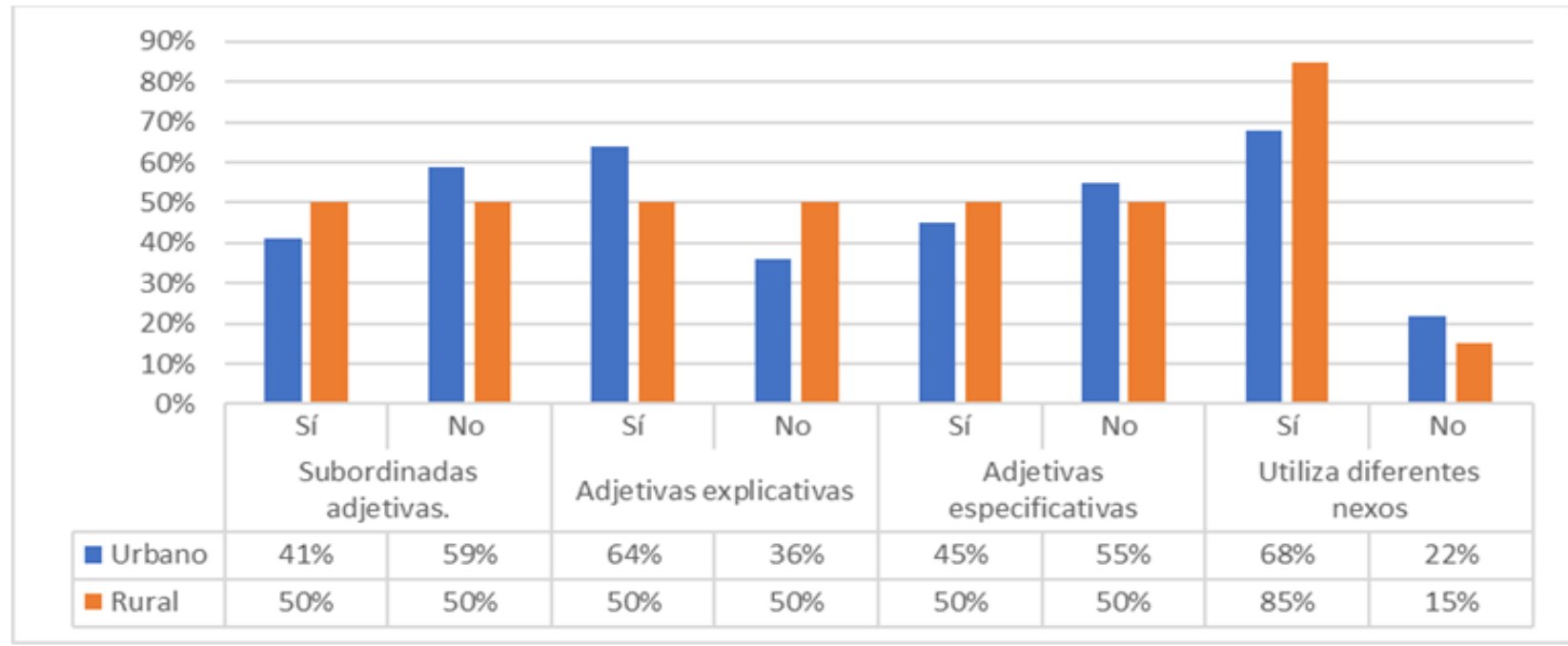

Gráfico $\mathrm{N}^{\circ}$ 13. Cuestionario utilizado para la revisión del texto diagnóstico. Fuente: tabla de análisis, ver anexo No3.4.

La información contenida en los gráficos $\mathrm{N}^{0} 11,12$ y 13 , es de importancia para este estudio: la oración subordinada adjetiva. Esta se construyó con los aportes del cuestionario (encuesta). Los porcentajes de afirmación de las variables masculino 50\%, privado 67\% y rural $50 \%$ están en el nivel medio, por lo tanto, el porcentaje de negación tiene resultados similares. Esto indica que los discentes no se habían apropiado adecuadamente de este conocimiento, porque no se evidencia de forma positiva en sus respuestas.

En el segundo y tercer aspecto de los gráficos $\mathrm{N}^{\circ} 11,12$ y 13, se les consultó sobre el tipo de oración subordinada utilizada en la escritura del texto diagnóstico. Los porcentajes del "Sí" para el segundo: masculino $90 \%$, privado $83 \%$ y urbano $64 \%$; en la tercera femenino $53 \%$, público $50 \%$ y rural $50 \%$. Lo anterior indica, que la muestra está dividida, el primer grupo de porcentajes asegura utilizar la subordinada adjetiva explicativa y el segundo la adjetiva especificativa. Los discentes respondieron a este cuestionario usando sus conocimientos previos, es por eso la existencia de esas respuestas, lo que sigue demostrando la desvinculación que se han formado de la gramática con la expresión escrita.

El último aspecto consultado en el cuestionario y presentado en los gráficos $\mathrm{N}^{\mathrm{o}} 11,12$ y 13, es el relacionado con el uso de diferentes nexos para insertar la oración subordinada adjetiva. A los discentes, se les preguntó sí usaron los nexos siguientes: que, cual, donde, cuando y quien. Las variables femenino $78 \%$, privado $83 \%$ y rural $85 \%$, indican que los discentes estaban convencidos de utilizar esos nexos para insertar la subordinada adjetiva. Sin embargo, en los casos analizados se evidencia que el de mayor uso es "que" y pocas veces "cual". 
Fallas estructurales de la oración subordinada adjetiva...

Escobar Soriano / Sandoval

TDA13

El femicidro. Considero ques un acto que puede darse por varias lrazones, oin embargo son racones no validas puesto que nadie leuede, ni debería privar de ta vida a

TDA42

En Nicaragua es un caso que se esta gemerando pero no thay que quedarnus in stends, debemos hablar este problemo ante la sociedad. pars que ast se hagr justicia. No mas violendia hado la mujer. "Alto al femicidio.

TDA1

de Tratar ester gobierno ha i do mejorando de violencias hai más - portunidad de denunciar, menos corrupción hasta un apoyo como es Mefamilia institución que da a compañamiento a la mujer a la denuncia con la policia.

TDA3

u las myeres, por es se llew lamuerte Pero kay leyes

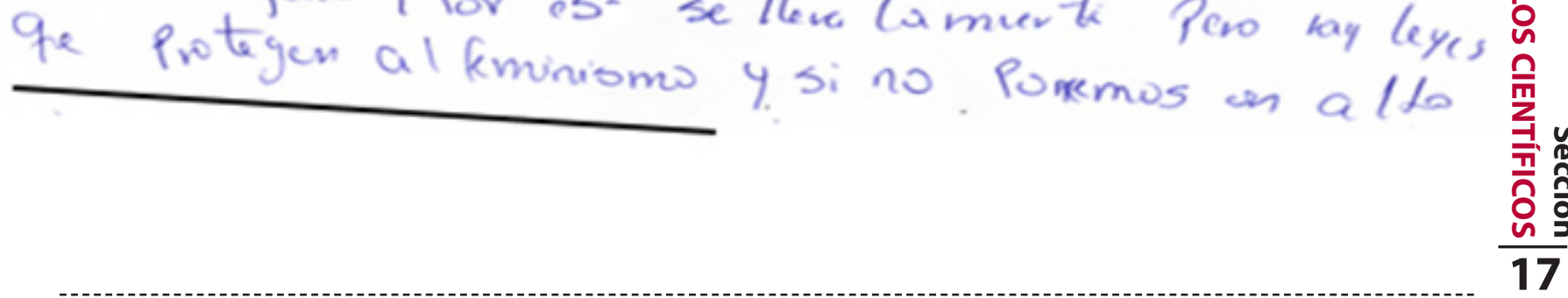




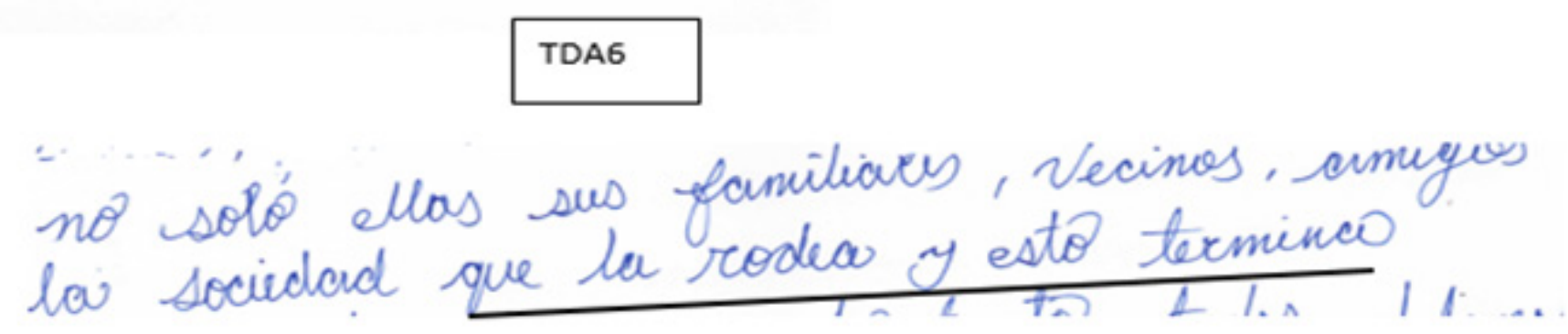

Se observará en textos seleccionados de la muestra, los cuatro aspectos abordados en los gráficos $\mathrm{N}^{0}$ 11, 12 y 13. En los textos TDA13, TDA42, TDA1, TDA3 y TDA6 se explica las situaciones relacionadas con los gráficos 11, 12 y 13. La primera consiste en el uso de la subordinada adjetiva en el texto diagnóstico; efectivamente en los ejemplos se observa el uso de la subordinada, la que domina la escritura, pero esto no se refleja en el porcentaje de los gráficos.

El segundo y tercer aspecto, se refiere al tipo de subordinada adjetiva utilizado en el texto diagnóstico argumentativo. En los gráficos ya mencionados, los porcentajes tienen un balance, entre la explicativa y la especificativa. Sin embargo, en los dos textos ejemplos (TDA13, TDA42, TDA1, TDA3 y TDA6), el tipo de subordinada utilizado en los escritos son de tipo especificativa. Además, se constató en otros textos, que el uso de subordinada adjetiva explicativa fue mínimo.

El cuarto aspecto abordado en los gráficos $N^{0} 11,12$ y 13, está relacionado al nexo utilizado para insertar la subordinada adjetiva. Los estudiantes indicaron utilizar una variedad, pero en los textos ejemplo TDA13, TDA42, TDA1, TDA3 y TDA6 el nexo usado es "que". Este tipo de nexo, está presente en la mayoría de los casos analizados y no se produjo el uso de diversos nexos, tal como los discentes lo establecieron en el cuestionario.

Se procedió al análisis detallado de la oración subordinada adjetiva presente en el texto diagnóstico, del cual se establecería las dificultades en los esquemas sintácticos subordinados adjetivos. Esto permitió determinar los errores en la construcción de la oración subordinada adjetiva de tipo: explicativa o especificativa, las cuales limitan la progresión de las ideas en el texto.

Con el resultado se construyó un gráfico, en el cual se refleja los porcentajes que indica los errores en el uso de la subordinada adjetiva presente en los textos: 


\begin{tabular}{|c|c|c|c|c|c|c|}
\hline \multirow{3}{*}{$\begin{array}{r}150 \% \\
100 \% \\
50 \% \\
0 \%\end{array}$} & & & & & & \\
\hline & & & & & & \\
\hline & Femenino & Masculino & Público & Privado & Urbano & Rural \\
\hline - Fallas en la explicativa & $3 \%$ & $0 \%$ & $3 \%$ & $0 \%$ & $5 \%$ & $0 \%$ \\
\hline Fallas en la especificativa & $97 \%$ & $90 \%$ & $97 \%$ & $100 \%$ & $95 \%$ & $100 \%$ \\
\hline
\end{tabular}

Gráfico N. ${ }^{\circ}$ 14. Errores en el uso de la oración subordinada adjetiva: explicativa y especificativa.

En el gráfico $\mathrm{N}^{\circ} 14$, se hace un resumen cuantificable de la cantidad de estudiantes que fallaron en el uso de la oración subordinada adjetiva: explicativa y especificativa. Los porcentajes en los errores mostrados en el uso de la subordinada especificativa son los más altos. Es este tipo de oración es la más utilizada en los textos. Los estudiantes desconocen la explicativa. Esto es una limitante para el estudiantado, quienes desconocen de manera práctica el uso de este recurso lingüístico, propuesto por la gramática.

\begin{tabular}{|c|c|c|c|}
\hline \multirow{5}{*}{$\begin{array}{r}80 \% \\
60 \% \\
40 \% \\
20 \% \\
0 \%\end{array}$} & & & \\
\hline & & & \\
\hline & & & \\
\hline & & & \\
\hline & $\begin{array}{l}\text { Acierto en el uso de la } \\
\text { subordinada adjetiva. }\end{array}$ & $\begin{array}{c}\text { Acierto a medias en el } \\
\text { uso de la subordinada } \\
\text { adjetiva }\end{array}$ & $\begin{array}{c}\text { Desaciertos en el uso } \\
\text { de la subordinada } \\
\text { adjetiva }\end{array}$ \\
\hline Explicativa & $5 \%$ & $0 \%$ & $0 \%$ \\
\hline Especificativa & $0 \%$ & $24 \%$ & $71 \%$ \\
\hline
\end{tabular}

\section{Gráfico $\mathrm{N}^{\circ} 15$. Uso de la oración subordinada adjetiva: explicativa y especificativa.}

El gráfico $\mathrm{N}^{0} 15$, muestra el resultado sobre el uso de la subordinada adjetiva. La primera categoría presenta los aciertos en el uso de la subordinada adjetiva, según los tipos. En ese sentido, la subordinada adjetiva de tipo explicativa es la que presenta $5 \%$ de acierto. Un dato interesante, porque es la que tiene menos uso y es la de registro acertado. En cambio, los aciertos a media y desaciertos ocurrieron en la subordinada adjetiva de tipo especificativa, además, esta es la de mayor uso.

Se utilizarán diferentes textos, para mostrar lo presentado en el gráfico № 15 . El primer aspecto de interés es presentar los aciertos en el uso de la subordinada adjetiva. Para el análisis se utilizaron los parámetros establecidos en el sistema categorial: 
El texto TDA 21 es un ejemplo del acierto, en el uso de la subordinada adjetiva, por parte del estudiante. En él se identifica la subordinada de tipo explicativa, la que fue insertada mediante el nexo simple "que", además el uso de la coma también indica la presencia de esta. Se califica de acertada por el hecho de que tiene un antecedente y consecuente. Entre ellos, tiene una función sintáctica y mantienen relación anafórica. No obstante, la redacción presenta redundancia de ideas por la repetición de la palabra inicio y falta de concordancia con el consecuente (inicie).

TDA 21

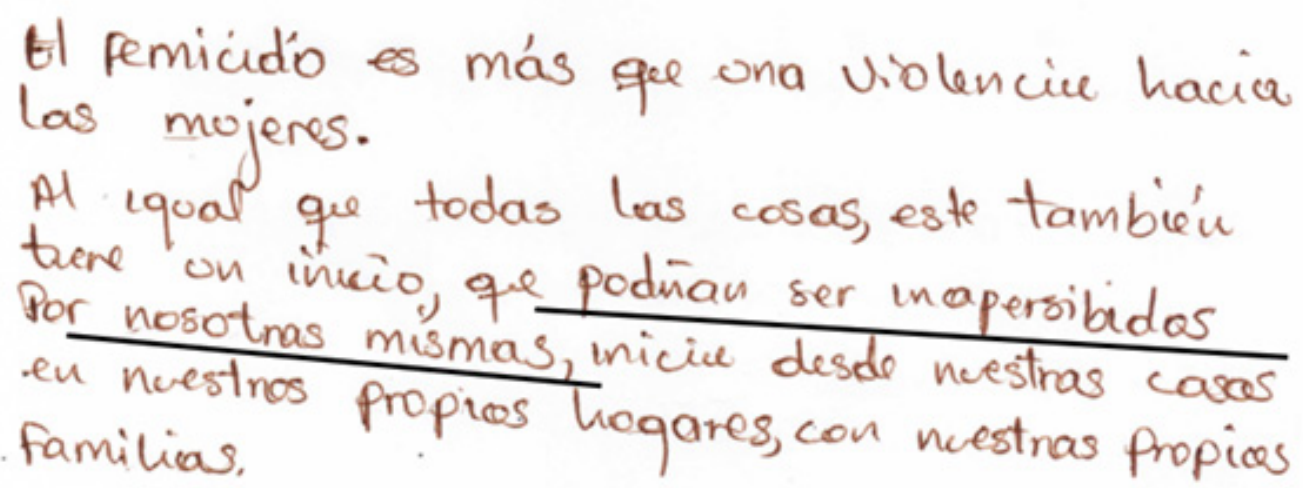

En el párrafo tomado del texto TDA42, se observa el uso de los relativos "lo que", "que" y "el cual". Estos cumplen con la función de introducir la subordinada adjetiva. El primero, inserta una adjetiva de tipo explicativa, la cual contiene información adicional y contribuye a la progresión de las ideas. El segundo, inserta una adjetiva de tipo especificativa y cumple con la relación entre el antecedente y el consecuente. Pero, en la tercera oración hay una falla de concordancia entre el nexo y el consecuente. Esto provoca la desvinculación entre las ideas, lo que contribuye a crear un texto inconexo, por lo tanto no ocurrirá la progresión temática.

\section{TDA42}

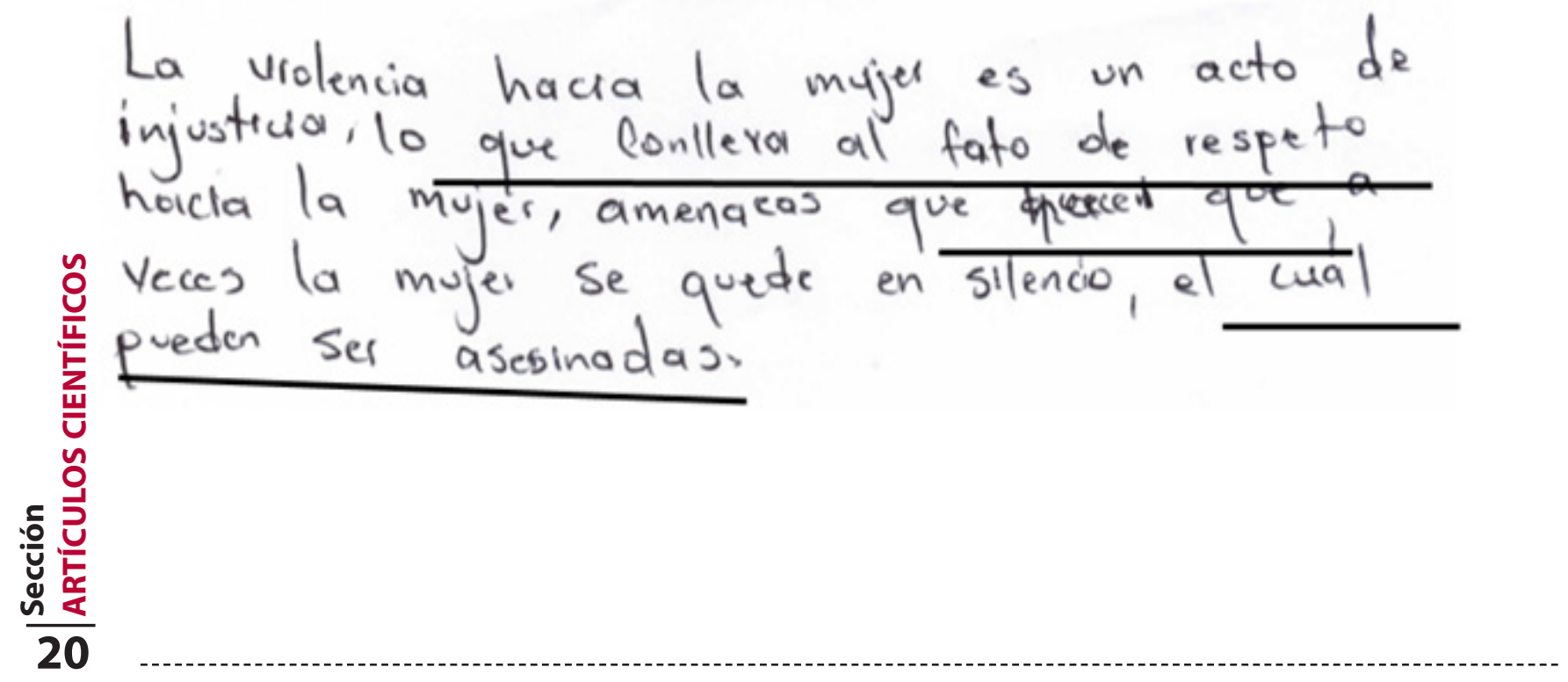


En el texto, cuyo código es TDA 32, se identifica el uso del relativo simple “que”. Este inserta una subordinada de tipo explicativa. El error se produce, porque en sí la información de la subordinada tiene relación con el antecedente, pero no con el consecuente. Los enunciados del párrafo carecen de coherencia, por lo tanto, la desvinculación de las ideas afecta la unidad del texto. Esto demuestra que los estudiantes tienen ideas para exponer en el texto, las cuales no logran concretar en ideas coherentes y cohesionadas, porque no ordenan las ideas en oraciones y estas enlazadas mediante conectores.

TDA32

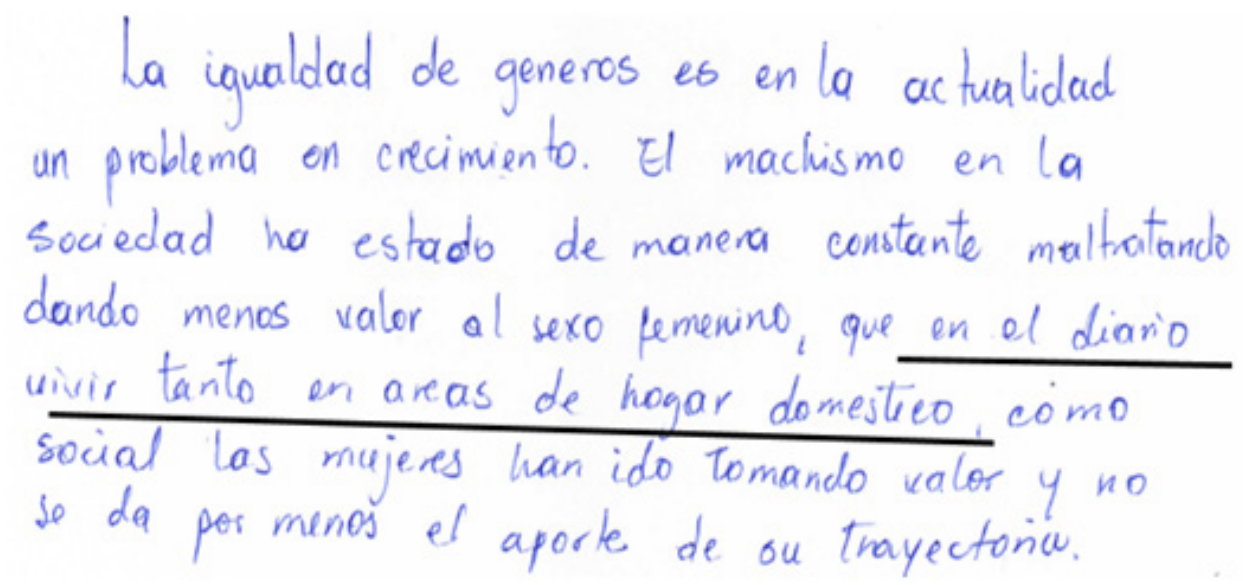

En el texto TDA 1, se identifica el uso de la subordinada adjetiva especificativa. Esta oración se introdujo mediante el nexo simple "que"; pero, el error se presenta entre la subordinada y el consecuente, porque se rompe la relación llamada anafórica, entre ellos. Por eso, se afecta la coherencia entre las ideas.

\section{TDA 1}

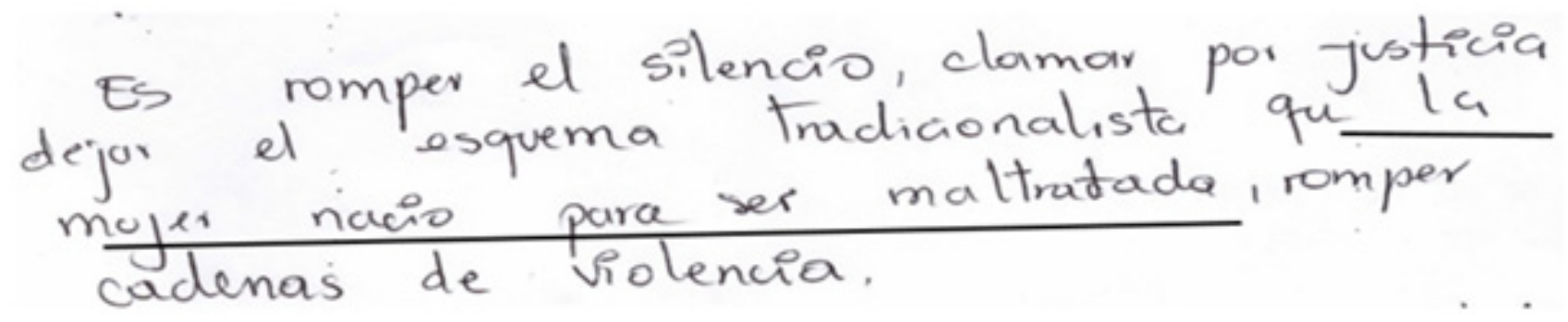

En el texto TDA6 está presente el uso de la subordinada adjetiva especificativa. Introducida por el nexo simple "que", este tiene relación directa con el antecedente, pero no con el consecuente. Esto se debe, claro está, al poco dominio de la gramática, la cual influye desde la construcción de las oraciones hasta el uso de los signos de puntuación. 
TDA 6

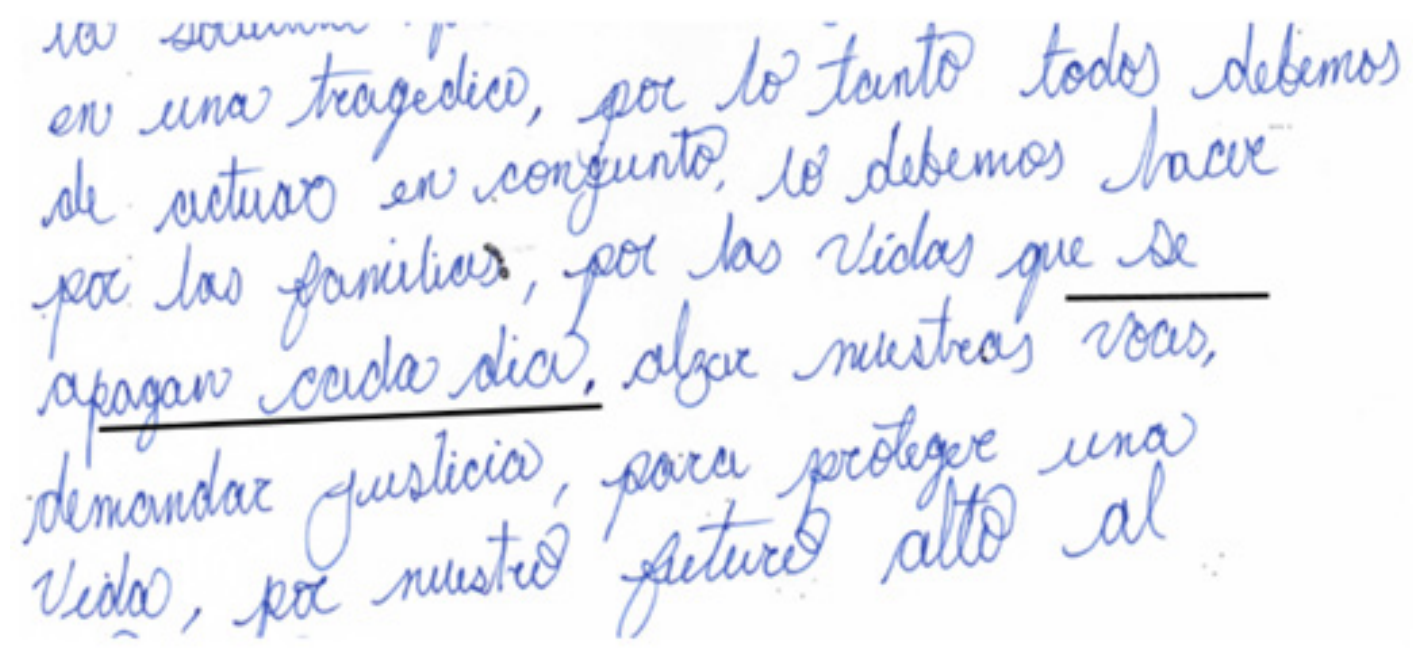

Estos conocimientos se forman a lo largo de la vida académica (primaria- secundaria). Producto del poco dominio teórico-práctico formado en esa etapa de la vida, se han proliferado problemas en los discentes, los que se manifiestan en la escritura. Por eso, el estudiante debe adquirir de forma consciente el uso y manejo de los elementos de su lengua natal, para su aplicación en diferentes contextos.

En el texto TDA 13, se analiza el uso de la oración subordinada adjetiva especificativa. Esta fue insertada mediante el relativo simple "que"; pero, en el párrafo se produjo un abuso del nexo, no permite una adecuada progresión de las ideas.

\section{TDA 13}

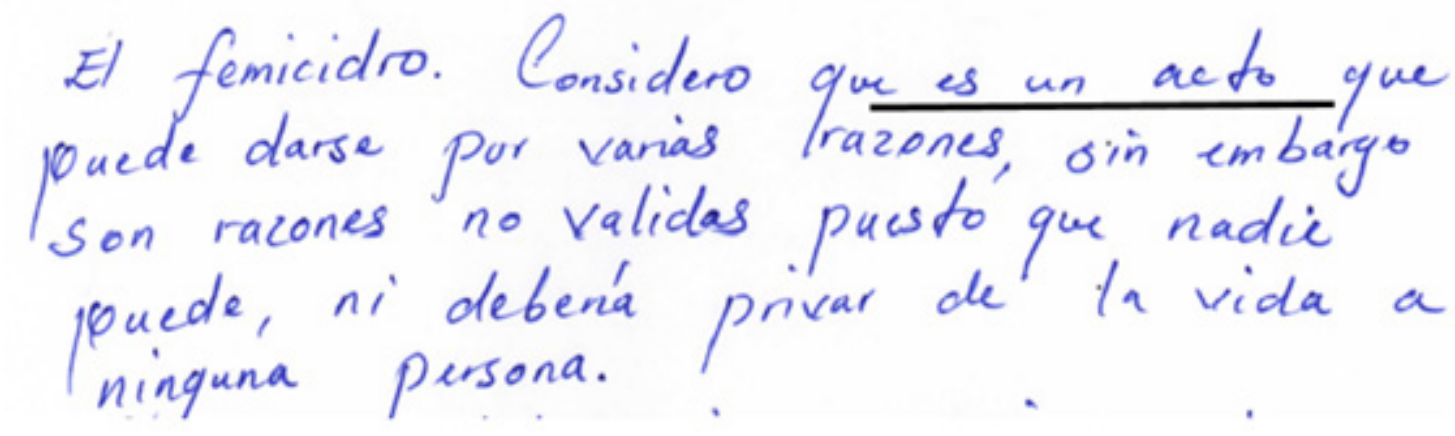

El texto TDA 29 muestra las dificultades enfrentadas por su escritor. Estas inician en el sujeto (justicia), sobre el que recae toda la acción del párrafo. Las formas verbales conjugadas (enfoca, hacia, tendrá) utilizadas en el escrito no favorecen la redacción de las ideas. Por otra parte, se detecta el uso de la subordinada adjetiva de tipo especificativa, la cual es introducida por el nexo "que". La falla en la construcción consiste en la falta de relación entre el antecedente el nexo y el consecuente. 


\section{TDA 29}

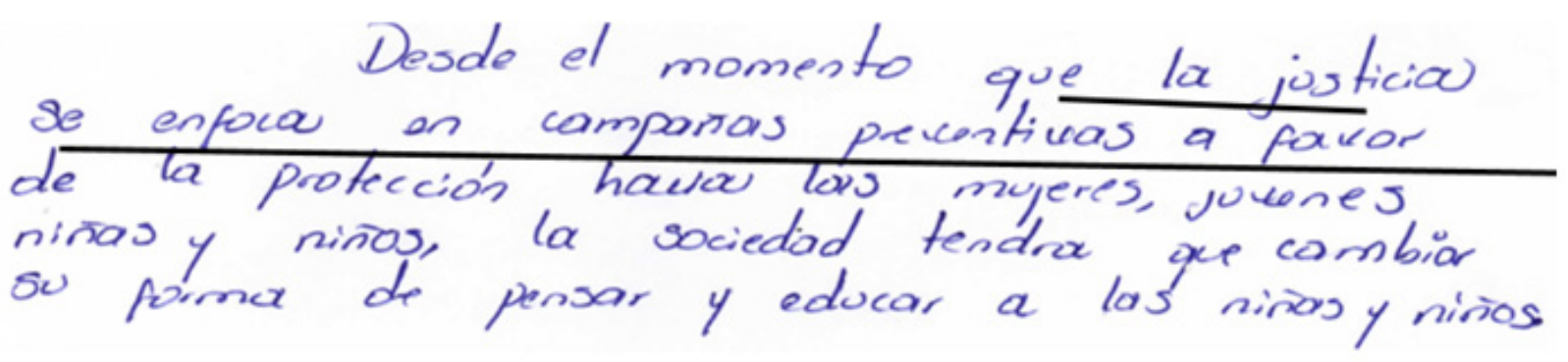

En el texto TDA48, el desorden en la construcción de las ideas no favorece la transmisión de la información. Esa problemática es producto del uso de la sintaxis de la lengua oral en la escrita. Eso, contribuye con las fallas en la subordinada adjetiva. Porque las estructuras de escritura son producto del proceso de meditación, en cambio las de lengua oral son espontáneas. Lo último es lo evidente en el texto, lo que causa la falla en la subordinada adjetiva, porque no hay relación con el consecuente.

\section{TDA 48}

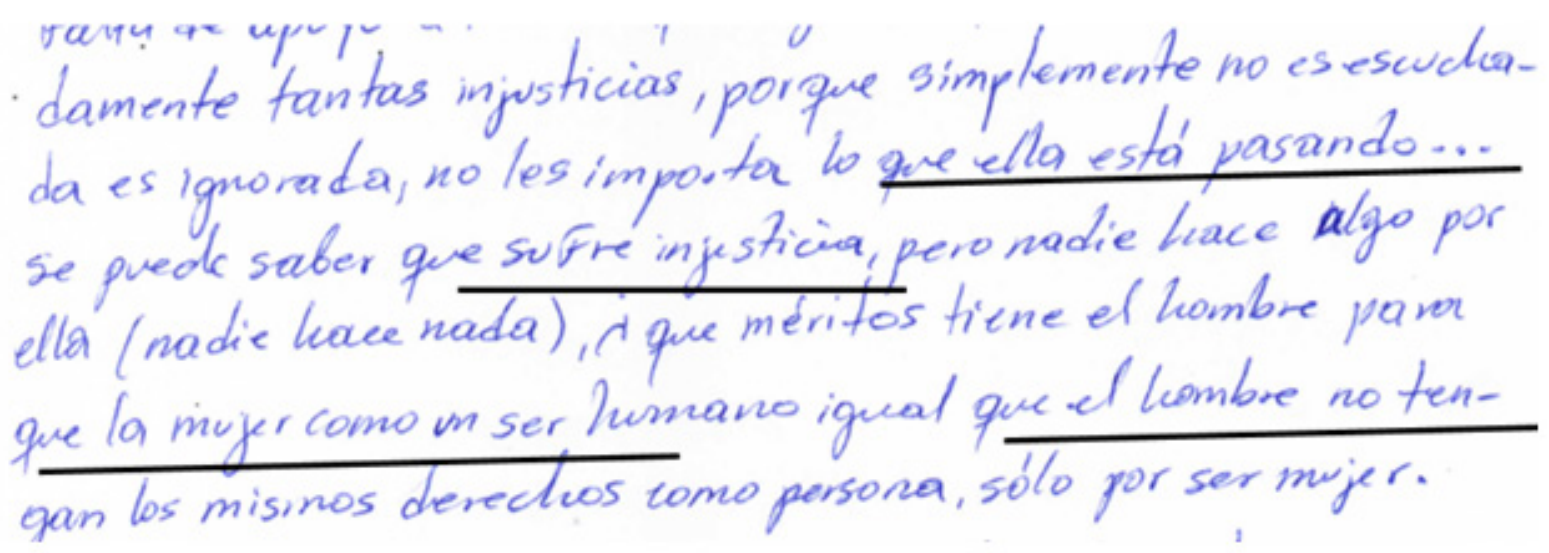

Esta falla se agudiza, porque no se ve dónde termina el enunciado. El discente construyó una cadena de enunciados compuestos. Por lo anterior, al revisar el antecedente inmediato de cada oración subordinada se aprecia que no está bien establecida la relación entre ellos y además, al perderse la conexión con el antecedente, también se pierde con el consecuente.

En el texto (TDA 32), se observa el uso de una construcción subordinada. A simple vista, parece ser una oración subordinada adjetiva explicativa. Sin embargo, el detalle más sobresaliente es que al observar su construcción con detenimiento, se aprecia que la forma verbal conjugada no concuerda con el antecedente en número y persona. Es decir, se introduce la oración, pero esta tiene un error de concordancia. Esta anomalía es causa de errores en dos vías: en la estructura de la subordinada y en la coherencia del párrafo. Pero realmente las subordinadas identificadas son de tipo objeto directo. 


\section{El Femicidio.}

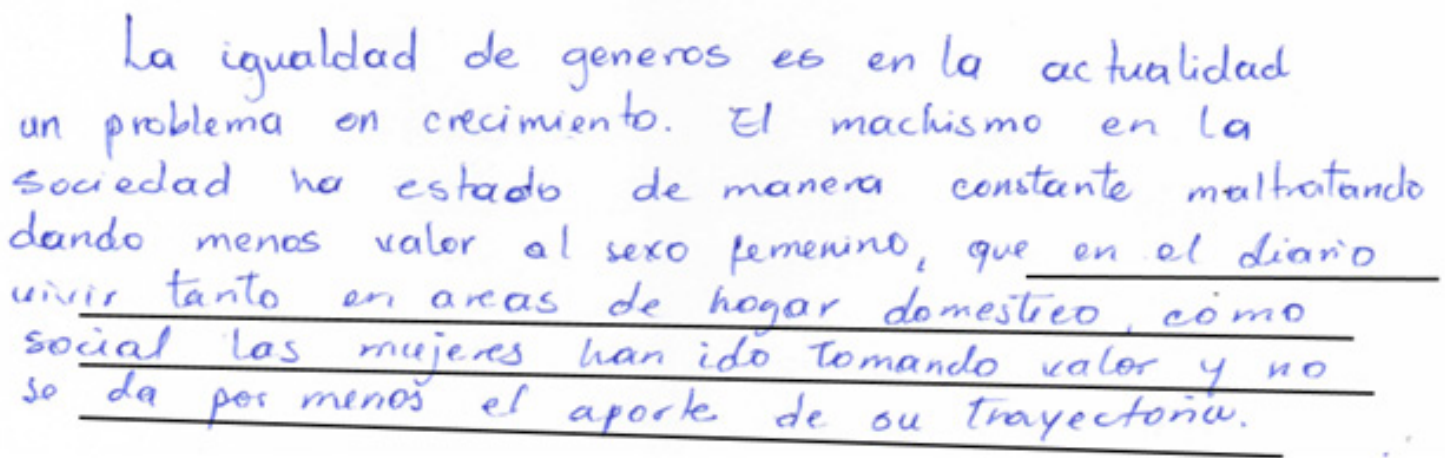

El texto TDA42, muestra la utilización de los nexos "lo que"y "el cual". Los dos corresponden al uso de relativos complejos en la construcción de la subordinada adjetiva. Las dificultades radican en que efectivamente introducen una subordinada adjetiva de tipo explicativa con el nexo "lo que", pero, dentro de esta misma ubica otra subordinada especificativa (que hace que). A esta falta se le conoce como queísmo. Por lo tanto, no se cumple con la estructura ideal de la subordinada. Con el nexo "el cual", ocurre que no hay concordancia de persona y número con el antecedente.

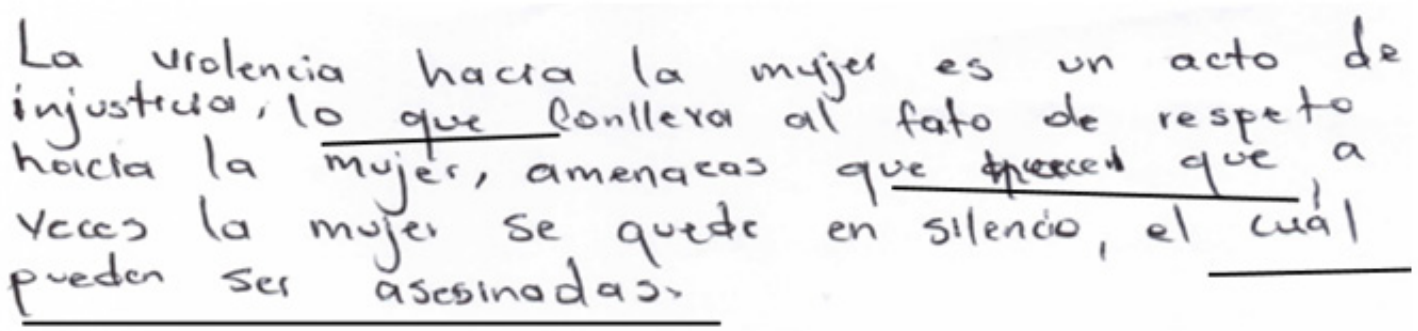

En resumen, el texto presenta proposiciones unidas por comas, pero no cumplen con la estructura de una oración subordinada adjetiva explicativa, ni la primera ni la segunda. Esto parece producto de la influencia de la lengua oral. Claramente esta construcción utilizada en el anterior texto (TDA42) no cumple con la función indicada. Por tanto, no aporta a una restricción, precisando la denotación del grupo nominal al cual pertenece.

El texto TDA3 muestra la reiteración del uso del relativo simple "que", en el intento de elaborar un párrafo, sin emplear la forma idónea para la construcción de la oración subordinada adjetiva especificativa. Por lo tanto, las fallas se enfocan, en la falta de relación entre el antecedente, nexo y el consecuente, actuando cada uno como elementos independiente dentro del párrafo, evitando la jerarquía entre las ideas y la progresión temática. 


\section{TDA 3}

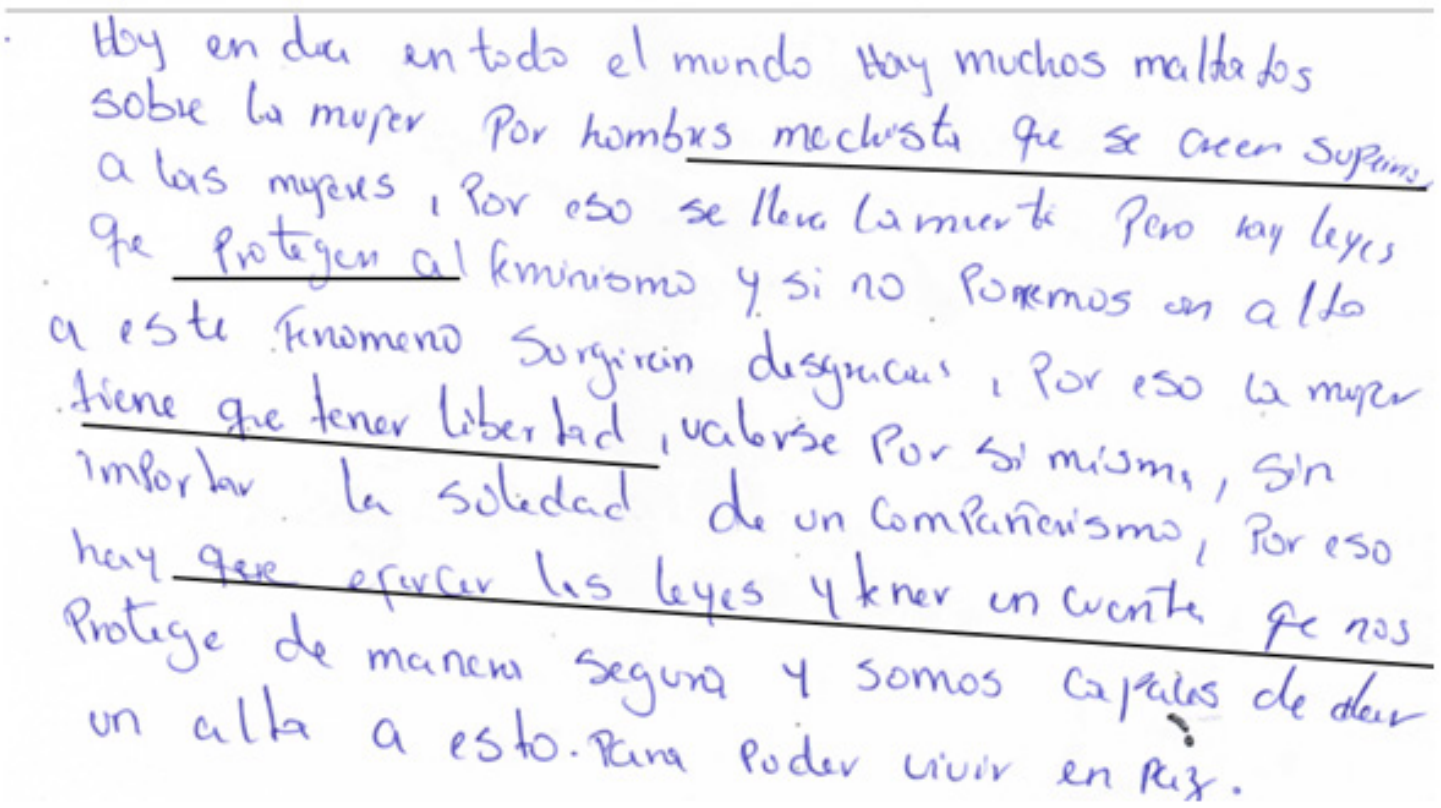

Los beneficios del uso de la gramática son incontables, como su presencia en la expresión escrita. Pero, la falta de dominio de la teoría y la incapacidad de los estudiantes para utilizarla en la práctica, les impide desarrollar todo su potencial. Por lo anterior, es el tiempo de que las instituciones e instancias correspondientes asuman un papel más beligerante en la enseñanza de la expresión escrita.

\section{CONCLUSIÓN}

Los estudiantes demostraron desconocer la importancia de la gramática para la escritura, porque cerca del 50\% afirmaron que no estaba presente en el escrito y similar porcentaje dijo no utilizarla para escribir algún texto. En el aspecto de la oración subordinada adjetiva se constató que la de mayor uso es la especificativa y el nexo simple "que", estos son los de mayor presencia en los textos diagnósticos. Por otra parte, la subordinada adjetiva explicativa y los nexos complejos son los que tienen menor presencia en los textos. Por eso, se puede afirmar que, en el texto diagnóstico argumentativo, se comprobó errores en alto porcentaje en la construcción de las oraciones subordinadas adjetivas (ambos tipos). Por último, en el análisis se hizo considerando diferentes variables: sexo (femenino-masculino), centro de procedencia (público-privado) y lugar de procedencia (urbano-rural), sin embargo, todos presentaron las mismas dificultades en dominio del proceso de composición y fallas en la construcción de la subordinada adjetiva. 


\section{REFERENCIAS}

Arzaluz, S. (2005). La utilización del estudio de caso en el análisis local. El Colegio de Sonora. México. Recuperado de: http://www.redalyc.org/articulo. oa?id=10203204

Chadwick, C. (1999). La psicología del aprendizaje desde el enfoque constructivista. Revista Latinoamericana de Psicología, vol. 31, núm. 3 pp. 463-47 Fundación Universitaria Konrad Lorenz Bogotá, Colombia. Recuperado de: http://www. redalyc.org/pdf/805/80531303.pdf

Giammatteo, M. (2013) “Por qué y para qué enseñar gramática? La gramática en la formación de habilidades cognitivolingüísticas”, SIGNOS ELE, 7, diciembre 2013, URL http://p3.usal.edu.ar/index. php/ele/article/view/2003, 16 págs.

Hernández,S.(2008).Elmodeloconstructivista con las nuevas tecnologías: aplicado en el proceso de aprendizaje. En: Comunicación y construcción del conocimiento en el nuevo espacio tecnológico [monográfico en línea]. Revista de Universidad y Sociedad del Conocimiento (RUSC). Vol. 5, n. ${ }^{\circ}$ 2. UOC. [Fecha de consulta: 20/10/2015].

http://dialnet.unirioja.es/servlet/ articulo? codigo $=2799725$

Luzón, J. y Soría, I. (1999). El Enfoque Comunicativo en la Enseñanza de Lenguas. Un Desafío para los Sistemas de Enseñanza y Aprendizaje Abiertos y a Distancia. Instituto Cervantes (España)
Meneses Báez, A. L., Mata, F. S., \& Ravelo Contreras, E. L. (2007). Description of cognitive processes involved in essaywriting. Acta Colombiana de Psicología, 10(1), 83-98.

Nueva gramática de la lengua española (2010). Asociación de academias de la lengua española.

Piaget, J. (s. f.). Seis estudios de psicología. Recuperado el 26 de junio del 2015

Rodríguez, C. (2012). La enseñanza de la gramática: las relaciones entre la reflexión y el uso lingüístico. Aceptado: 26/06/12 Revista iberoamericana de educación. n. ${ }^{\circ}$ 59, pp. 87-118 (1022-6508) - oei/caeu

Rodríguez, M. y Moreira, M. (2002). Modelos mentales vs esquemas de célula investigações em ensino de ciências - v7 (1), pp. 77-103.

Torres, R. M. (2004). Comunidad de aprendizaje. La educación en función del desarrollo local y del aprendizaje.

Vivas, N. (2010). Estrategias de aprendizaje, Gondola issn 2145-4981 vol. 5 no. pp 2737 